\title{
Quantum Standpoint for a More Understandable Universe
}

\author{
Sebastiano Tosto
}

ENEA Casaccia, Via Anguillarese 301, 00123 Roma, Italy

\begin{abstract}
The study implements the concept of space-time quantum uncertainty to outline a possible model of universe. Simple considerations show that the relativistic equations inferred as a consequence of the uncertainty allow describing effects typically expected in the frame of quantum phenomena, e.g., the fluctuations. These results contribute to clarify issues today still open, like the dark energy, the inflationary expansion of the universe and the possible existence of antigravity.
\end{abstract}

Keywords: Quantum Theory, Quantum Phenomena, Multidisciplinary Science

\section{INTRODUCTION}

The cosmology is a multidisciplinary science that involves all branches of the theoretical physics. Its modern formulation was born with the introduction of the cosmological constant into the general relativity; then, after the early De Sitter model, its successive development was closely connected to the concurrent progress of the quantum theory.

The multidisciplinary basis of the cosmology, compelled by the necessity of investigating quantumand macro-scale phenomena in the universe, e.g., the nuclear reaction driven abundance of chemical elements and the formation of black holes, poses a wide variety of conceptual issues.

The major theoretical challenges concern at present: the dark energy and the missing mass (Trimble, 1987; Bertone et al., 2005; Kroupa, 2010), the matterantimatter asymmetry (Canetti et al., 2012; Dine and Kusenko, 2003; Dolgov, 2002; Zhi-Zhong, 2007), the growth mechanism and final fate of the universe (Adams and Laughlin, 1997; Krauss and Starkman, 2000; Islam, 1977; Carroll and Chen, 2004; Liddle and Lyth, 2000). To these very general topics contribute also more specific subjects, e.g., the element abundance formation (Burbridge et al., 1957), the black hole physics (Davies, 1978; Penrose, 1965; Hawking and Penrose, 1970) and the quantum theories of gravity (Gupta, 1962; Weinberg,
1996). Primary tools to tackle these issues are the quantum theory and the general relativity, whose connection represents itself an outstanding crucial point of the modern physics. Despite the huge number of papers published on these topics, some questions are still open; for instance, just the way of bridging quantum theory and relativity is still actively explored and debated.

The physical models of universe play a special role in cosmology, mostly because the experimental data are accessible in a limited domain of space and time consistent with the light speed. In general the experimental data corroborate the theoretical model, which usually implements also results of other measurements required by its formulation; often however in the science of the universe this is impossible for practical reasons, so the reliability of a theoretical model rests on its self-consistency and ability to provide itself sensible estimates of missing experimental data.

A paper recently published (Tosto, 2013) introduces a theoretical model that implements quantum concepts to propose possible answers to some among the aforesaid open points; the input data of the model were the acknowledged values of radius $r_{u}=4.4 \times 10^{26} \mathrm{~m}$, age $r_{u}=4.3 \times 10^{17} \mathrm{~s}$ and mass $\mathrm{m}_{\mathrm{u}}=$ $3 \times 10^{52} \mathrm{~kg}$ of today's universe. It is crucial the fact that $m_{u}$ accounts for the mass of visible stars only.

The present paper extends the outcomes exposed in (Tosto, 2013) with further considerations on the space- 
time quantum uncertainty; this standpoint helps to clarify some issues previously quoted and enhances our understanding of the universe.

\subsection{Quantum Background}

Consider a set of quantum particles forming a complex system. Whatever the specific nature of these particles and their interaction might be, the statistical formulation of the quantum uncertainty:

$$
\Delta \varepsilon \Delta \mathrm{t}=\mathrm{n} \hbar=\Delta \mathrm{p}_{\mathrm{x}} \Delta \mathrm{x}
$$

Is regarded as fundamental principle to describe the properties of the system; $\mathrm{n}$ is the number of quantum states accessible to any particle of the set. The second equality holds with the same number of states because $\Delta \mathrm{p}_{\mathrm{x}}$ and $\Delta \mathrm{x}$ are formally inferred from $\left(\Delta \varepsilon / \mathrm{v}_{\mathrm{x}}\right)\left(\mathrm{v}_{\mathrm{x}} / \Delta \mathrm{t}\right)$, being by definition $\mathrm{v}_{\mathrm{x}}=\Delta \mathrm{x} / \Delta \mathrm{t}$ an arbitrary velocity; the right hand side notation symbolizes any couple of conjugate dynamical variables. The basic idea motivating the Equation (1) is that the local values of the dynamical variables of every particle in the system are perturbed in a complex way by mutual interactions and thus change within appropriate range of values. This suggests the chance of discarding the local dynamical variables conceptually and not as a sort of approximation to simplify some calculation, in agreement with the quantum impossibility of knowing exactly both conjugate dynamical variables. The present model introduces since the beginning uncertainty ranges that replace the local dynamical variables of every particle in the system; the greater the sizes of the former, the wider the spectrum of values allowed to the latter. The Equation (1) represent the quantum constrain and contextually the only kind of information able to balance this agnostic assumption. No hypothesis is necessary about the uncertainty range sizes, which are arbitrary by fundamental assumption; $\mathrm{n}$ is arbitrary too, it does not represent some specific number rather it symbolizes any number of allowed states. Regardless of the complexity of the system and kind of particles constituting it, this standpoint moves the physical interest from the actual conjugate dynamical variables of the particles in the system to the uncertainty ranges including all their possible values. The physical properties of the observables depend on the latter and not on the former, which are considered random, unknown and unpredictable; also, $\mathrm{n}$ coincides with the quantum number defining the allowed eigenvalues. Once renouncing to the concept of local time and space coordinates, the concept of velocity results indeterminate as well; it is only possible to say that $\Delta \mathrm{x} / \Delta \mathrm{t}$ defines an average value of velocity, which however is numerically indefinable like $\Delta \mathrm{x}$ and $\Delta \mathrm{t}$ themselves. Despite the standpoint proposed here is more agnostic than that based on the operator formalism of quantum mechanics, the results are however completely analogous; moreover the wave formalism appears to be a corollary of Equation (1). Some examples about how to exploit Equation (1) to calculate the eigenvalues of non-relativistic angular momentum and hydrogenlike electron energy levels are reported in (Tosto, 1996a); the approach then has been also exploited to treat many electron atoms and diatomic molecules (Tosto, 1996b). For brevity, details about the mathematical approach followed in these papers are omitted. It is significant instead the chance of extending the early quantum approach also to the relativity. Let Equation (1) be defined in an arbitrary reference system $\mathrm{R}$ and write $\Delta \mathrm{x}=\mathrm{x}_{2}-\mathrm{x}_{1}$; for instance $\mathrm{x}_{1}$ is defined with respect to the origin of $\mathrm{R}$ and controls thus the position of $\Delta \mathrm{x}$ in $\mathrm{R}$, whereas $\mathrm{x}_{2}$ controls the size of $\Delta \mathrm{x}$. If however $\mathrm{x}_{1}$ is unspecified and indeterminable, then $\mathrm{R}$ is also unspecified and indeterminable itself. This conclusion is nothing else but the well known postulate of relativity, according which all reference frames are equivalent to describe the physical systems. In fact Equation (1) hold in any reference system: whatever the transformation law of the range sizes between different $\mathrm{R}$ and $\mathrm{R}^{\prime}$ in reciprocal motion might be, the new equation $\Delta \varepsilon^{\prime} \Delta \mathrm{t}^{\prime}=\mathrm{n}^{\prime} \hbar=\Delta \mathrm{p}_{\mathrm{x}}^{\prime} \Delta \mathrm{x}^{\prime}$ is actually indistinguishable from (1). First of all $n$ and n' are in fact identical; whether referred to $\Delta \mathrm{xp}_{\mathrm{x}}$ or $\Delta \mathrm{x}^{\prime} \Delta \mathrm{p}_{\mathrm{x}}^{\prime}$, by assumption they represent all possible numbers of states and not an assigned number of states; so both denote anyway sets of integer numbers. Moreover, being the range sizes completely arbitrary, primed and unprimed equations are merely different notations of a unique uncertainty equation; in effect is unphysical to regard $\Delta \mathrm{x}$ and $\Delta \mathrm{x}$ ' as different extents of space delocalization once having acknowledged that neither size is actually specifiable. The input data of the present model, radius, age and mass of the universe, read accordingly Equation (2):

$$
\begin{aligned}
\Delta \mathrm{r}_{\mathrm{u}} & =4.4 \times 10^{26} \mathrm{~m} \\
\Delta \mathrm{t}_{\mathrm{u}} & =4.3 \times 10^{17} \mathrm{~s} \\
\mathrm{~m}_{\mathrm{u}} & =3 \times 10^{52} \mathrm{Kg}
\end{aligned}
$$


The next discussion highlights the relevance of this standpoint for the cosmological problems. A few examples of relativistic formulae inferred uniquely via Equation (1) are reported (i) because of their clarifying significance for the considerations exposed next and (ii) in order to make the present paper as self-contained as possible. A first important consequence appears clear since now. The relativity is classical physics in a 4D frame subjected to the covariance condition. If however the early Einstein equations of special relativity are entirely inferred in a quantum frame and accordingly regarded, then it must be true also their compliancy with all rules and weird features of the quantum world; e.g., they must agree with the existence of quantum fluctuations.

\subsection{Quantum Considerations and Relativity}

This section sketches shortly how the relativistic momentum and energy are obtained exploiting Equation (1) only. In an arbitrary delocalization range $\Delta \mathrm{x}^{(\mathrm{c})}$ defined in any $\mathrm{R}$ a photon travels at speed c; so Equation (1) $\operatorname{read} \Delta \mathrm{x}^{(\mathrm{c})} \Delta \mathrm{p}_{\mathrm{x}}^{(\mathrm{c})}=\mathrm{n}^{(\mathrm{c})} \hbar=\Delta \mathrm{t}^{(\mathrm{c})} \Delta \varepsilon^{(\mathrm{c})}$. The superscripts mention that the ranges are sized to fulfil the delocalization condition during an appropriate time range $\Delta \mathrm{t}^{(\mathrm{c})}$ : by definition $\Delta \mathrm{x}^{(\mathrm{c})} / \Delta \mathrm{t}^{(\mathrm{c})}=\mathrm{c}$, so $\mathrm{c} \Delta \mathrm{p}_{\mathrm{x}}^{(\mathrm{c})}=\Delta \varepsilon^{(\mathrm{c})}$. To find how the momentum and energy ranges $\Delta \mathrm{p}_{\mathrm{x}}^{(\mathrm{v})}$ and $\Delta \varepsilon^{(\mathrm{v})}$ of a massive particle travelling at rate $\mathrm{v}_{\mathrm{x}}<\mathrm{c}$ through the same $\Delta \mathrm{x}^{(\mathrm{c})}$ scale with respect to $\Delta \mathrm{p}_{\mathrm{x}}^{(\mathrm{c})}$ and $\Delta \varepsilon^{(\mathrm{c})}$, write $\Delta \mathrm{x}^{(\mathrm{c})} \Delta \mathrm{p}_{\mathrm{x}}^{(\mathrm{v})}=\mathrm{n}^{(\mathrm{v})} \hbar=\Delta \mathrm{t}^{(\mathrm{v})} \Delta \varepsilon^{(\mathrm{v})}$. The superscripts emphasize the new rate with which the particle travels the space range $\Delta \mathrm{x}^{(\mathrm{c})}$; neither $\mathrm{v}_{\mathrm{x}}$ nor $\mathrm{c}$ appear explicitly in this equation. The sets of integers represented by $n^{(v)}$ and $n^{(c)}$ describe all possible values allowed to $\Delta \mathrm{t}^{(\mathrm{v})} \Delta \varepsilon^{(\mathrm{v})}$ and to $\Delta \mathrm{t}^{(\mathrm{c})} \Delta \varepsilon^{(\mathrm{c})}$; yet, as previously sketched, the sets are physically are indistinguishable because anyway $\mathrm{n}^{(\mathrm{v})}=$ $\mathrm{n}^{(\mathrm{c})}=1,2,3, \ldots$ regardless of the specific range sizes or kind and travel rate of the particle. Hence it is possible to write $\Delta \mathrm{t}^{(\mathrm{v})} \Delta \varepsilon^{(\mathrm{v})}=\Delta \mathrm{t}^{(\mathrm{c})} \Delta \varepsilon^{(\mathrm{c})}$; this shows that $\Delta \mathrm{t}^{(\mathrm{c})}$ and $\Delta \varepsilon^{(\mathrm{c})}$ scale respectively like $\Delta \mathrm{t}^{(\mathrm{v})}=\left(\mathrm{c} / \mathrm{v}_{\mathrm{x}}\right) \Delta \mathrm{t}^{(\mathrm{c})}$, as it is reasonable and $\Delta \varepsilon^{(\mathrm{v})}=\left(\mathrm{v}_{\mathrm{x}} / \mathrm{c}\right) \Delta \varepsilon^{(\mathrm{c})}$ as a consequence. Replacing these positions in the previous equation, $\Delta \mathrm{x}^{(\mathrm{c})} \Delta \mathrm{p}_{\mathrm{x}}^{(\mathrm{v})}=\Delta \mathrm{t}^{(\mathrm{c})}\left(\mathrm{v}_{\mathrm{x}} / \mathrm{c}\right) \Delta \varepsilon^{(\mathrm{v})}$ yields $\Delta \mathrm{p}_{\mathrm{x}}^{(\mathrm{v})}=\Delta \varepsilon^{(\mathrm{v})} \mathrm{v}_{\mathrm{x}} / \mathrm{c}^{2}$. This result means that to any random local variable $p_{x}^{(v)}$ included in the range $\Delta \mathrm{p}_{\mathrm{x}}^{(\mathrm{v})}=\mathrm{p}_{\mathrm{x} 2}^{(\mathrm{v})}-\mathrm{p}_{\mathrm{x} 1}^{(\mathrm{v})}$ corresponds the random local variable $\varepsilon \mathrm{v}_{\mathrm{x}} / \mathrm{c}^{2}$ included in the range $\varepsilon_{2}^{(v)} v_{x} / c^{2}-\varepsilon_{1}^{(v)} v_{x} / c^{2}$. Actually the superscripts can be omitted, they have been introduced for clarity of exposition only and not to identify particular range sizes; being indeed both $\Delta \mathrm{p}_{\mathrm{x}}^{(\mathrm{v})}$ and $\Delta \varepsilon^{(\mathrm{v})}$ completely arbitrary like $\mathrm{v}_{\mathrm{x}}$ itself, the superscripts do not affect the functional relationship between the local random values of the respective variables, which therefore fulfil the condition:

$\mathrm{p}_{\mathrm{x}}=\varepsilon \frac{\mathrm{v}_{\mathrm{x}}}{\mathrm{c}^{2}}$

Regardless of how the respective uncertainty ranges are indicated. Moreover an identical reasoning holds in any other reference system R'; so one concludes that $\mathrm{p}_{\mathrm{x}}^{\prime}=\varepsilon^{\prime} \mathrm{v}_{\mathrm{x}}^{\prime} / \mathrm{c}^{2}$ is an invariant of special relativity. In principle the component of velocity defining the related momentum can be positive or negative depending on the direction of motion along the reference $\mathrm{x}$-axis; yet squaring this equation one surely handles positive terms. So write $\left(\mathrm{p}_{\mathrm{x}} \mathrm{c}\right)^{2}=\varepsilon^{2}\left(\mathrm{v}_{\mathrm{x}} / \mathrm{c}\right)^{2}$; since $\mathrm{v}_{\mathrm{x}}<\mathrm{c}$ for a massive particle one finds $\varepsilon^{2}>\left(p_{x} c\right)^{2}$, which compels writing $\varepsilon^{2}=\left(p_{x} c\right)^{2}+\varepsilon_{o}^{2}$. Calculate the limit $p_{x} / v_{x}$ for $v_{x} \rightarrow 0$; denoting this limit as:

$\lim _{\mathrm{v}_{\mathrm{x}} \rightarrow 0} \frac{\mathrm{p}_{\mathrm{x}}}{\mathrm{v}_{\mathrm{x}}}=\mathrm{m}=\frac{\varepsilon_{\text {rest }}}{\mathrm{c}^{2}}$

The concept of mass $\mathrm{m}$ is introduced as a consequence of the uncertainty, whereas Equation (3) yields $\lim _{v_{x} \rightarrow 0} \varepsilon=\varepsilon_{\text {rest }}$ in agreement with the idea that the limit must be finite; indeed no reason requires $\varepsilon \rightarrow 0$ for $\mathrm{v}_{\mathrm{x}} \rightarrow 0$. Thus $\mathrm{p}_{\mathrm{x}}=\mathrm{mv}_{\mathrm{x}}$ is the non-relativistic form of Equation (4), whereas the previous equation yields:

$\varepsilon^{2}=(\mathrm{pc})^{2}+\left(m c^{2}\right)^{2}$

Note that combining the Equation (3) and (5) one obtains the well known invariants of momentum and energy compliant with the Lorentz transformations. In relativity the local values of momentum and energy are exactly known; in Equation (3) they are random values falling within the respective uncertainty ranges, despite their functional relationship is of course identical. It is worth noticing that the connection between quantum mechanics and relativity concerned in (Tosto, 2012) is actually much more profound than that sketched here; the connection concerns indeed the basic postulates themselves of both theories, which merge therefore into the unique concept of space-time quantum uncertainty. 


\subsection{Quantum Mechanics, Newton Law and Black Holes}

Usually the origin of a black hole is associated to a collapse event, for instance when a large sized body with corresponding large mass shrinks to a new small sized body; this typically happens at the end of the life cycle of a massive star. However simple quantum considerations provide an alternative representation of a black hole with the help of Equation (1) only, which will be usefully exploited in the next section.

Let a free particle of mass $m$ be delocalized in the volume $\Delta \mathrm{l}^{3}$ : the average matter density $\rho=\mathrm{m} / \Delta \mathrm{l}^{3}$ exists in the volume of space where is delocalized the particle. The next considerations exploit the fact that the gravity constant $G$ times $\rho$ defines by dimensional reasons a reciprocal square time.

\subsection{Quantum Connotation of $G \rho=$ time $^{-2}$}

Regard first the reciprocal time as a frequency; then:

$\omega^{2}=\mathrm{Gm} / \Delta \mathrm{l}^{3}$

To explain the physical meaning of this result write the left hand side as $(\mathrm{n} \hbar \omega)^{2} /(\mathrm{n} \hbar)^{2}$ i.e., $(\Delta \varepsilon)^{2} /(\Delta \mathrm{p} \Delta \mathrm{l})^{2}$, where $\Delta \varepsilon$ is the energy range including all multiples of the ground energy $\varepsilon=\hbar \omega$ calculable with arbitrary values of $\mathrm{n}$. Moreover Equation (3) yields $\mathrm{p}=\hbar \omega \mathrm{v} / \mathrm{c}^{2}$; so, being $\omega \mathrm{v} / \mathrm{c}^{2}=2 \pi(\mathrm{v} / \mathrm{v})(\mathrm{v} / \mathrm{c})^{2}$ and $\mathrm{v} / \mathrm{v}=\lambda^{,-1}$, the result is $\mathrm{p}=\mathrm{h} / \lambda$ with $\lambda=(\mathrm{c} / \mathrm{v})^{2} \lambda$, This holds in general for electromagnetic waves in vacuum, $\lambda=\lambda$, and matter waves, $\lambda>\lambda$ '; indeed $\lambda^{\prime}, \mathrm{v}$ and $\omega$ are arbitrary. Equation (3) and (5) describe the particle as a corpuscle, $\varepsilon=\hbar \omega$ and $\mathrm{p}=\mathrm{h} / \lambda$ emphasize its wave behaviour; indeed the mass of the particle does not explicitly appear in these expressions of $p$ and $\varepsilon$. In this case, delocalizing any kind of particle in a volume of space means a wave of frequency $\omega$ propagating at rate $\mathrm{v}=\lambda^{\prime} \mathrm{v}$ in the given volume of space.

\subsection{Newtonian Connotation of $\mathrm{G} \rho=$ time $^{-2}$}

Regard now the left hand side of Equation (6) as a reciprocal square time range $\Delta \mathrm{t}^{-2}$; since $\Delta \mathrm{l} / \Delta \mathrm{t}$ has physical dimensions of velocity, G $\rho$ yields now $v^{2}=$ $\mathrm{mG} / \Delta \mathrm{l}$. Implement this equation multiplying and dividing the left hand side by an arbitrary mass km', with $\mathrm{k}= \pm 1$; one finds $\mathrm{U}=\mathrm{km}$ ' $\mathrm{mG} / \Delta \mathrm{l}$ with $\mathrm{U}=\mathrm{m}^{\prime} \mathrm{v}^{2}$; the energy $U$ describes thus the potential energy of an arbitrary test mass $\mathrm{m}$ ' in the gravitational field of $\mathrm{m}$ and fulfils the virial theorem $\mathrm{U}=2 \mathrm{~T}$ once regarding the quantities at both sides as average values. This is nothing else but the Newton law, which requires negative sign of $\mathrm{k}$; in principle, however, even a repulsive force with $\mathrm{k}=1$ is consistent with the quantum Equation (1). This means that anti-gravity is also possible and compatible with the present quantum frame. The fact that now $m$ instead of $\omega$ appears in this equation reveals nothing else but the well known wave/corpuscle dual behaviour of quantum particles.

\subsection{The Black Hole as a Corollary}

Exploit again $\Delta \mathrm{l} / \Delta \mathrm{t}=\mathrm{v}$ and $\mathrm{v}^{2}=\mathrm{Gm} / \Delta \mathrm{l}$. The fact that $\mathrm{v}$ is upper bounded defines a lower limit $\Delta \mathrm{l}_{0}=\mathrm{mG} / \mathrm{c}^{2}$ for $\Delta \mathrm{l}$ in the presence of a given mass $\mathrm{m}$; the length $\Delta \mathrm{l}_{0}$ results defined as a function of $\mathrm{G}$ and $\mathrm{m}$ only. Let $\Delta \mathrm{l}_{0}$ be the distance travelled by a photon starting from an arbitrary point, defined without loss of generality as the origin of the reference system R. Since the photon can move around the origin towards the negative or positive side of the reference axis with equal probability, indeed either sign of $\mathrm{v}$ is identically admissible, $\Delta \mathrm{l}_{\mathrm{o}}$ is one half of a total uncertainty range $\Delta \mathrm{l}_{\text {Schw }}$ where the photon is certainly enclosed; so $\Delta \mathrm{l}_{\text {Schw }}=2 \Delta \mathrm{l}_{0}$ yields:

$$
\Delta \mathrm{l}_{\text {Schw }}=2 \mathrm{G} \frac{\mathrm{m}}{\mathrm{c}^{2}}
$$

The interesting case of a photon orbiting around a black hole has been also described elsewhere. In conclusion quantum energy and momentum, Newton law and black hole condition are contextually inferred in a very elementary way as a corollary in the frame of a unique quantum equation, the space-time uncertainty of Equation (1). The important remark is that the black hole condition is obtained neither via gravitational collapse nor via escaping velocity from a gravitational mass, but examining the delocalization of a photon around a gravitational mass. Nevertheless remains true an outstanding feature: no photon can escape outside $\Delta \mathrm{l}_{\text {Schw }}$, which sets therefore an event horizon. Here this conclusion results because the boundary of the sphere of radius $\Delta \mathrm{l}_{\text {Schw }}$ centred on the starting point of a photon is defined just by the maximum distance travelled by that photon; otherwise stated no photon can trespass the boundary of a sphere having radius equal to the uncertainty range $\Delta \mathrm{l}_{\text {Schw }}$, which indeed includes all random positions allowed to the photon in conceptual 
agreement with the fundamental assumption underlying Equation (1). In this sense, even the light inside the black hole is confined within and defines itself the event horizon.

\subsection{Implications for Cosmology}

This section concerns the chance of describing the whole universe as a super-massive black hole, i.e., a universe whose total matter content fulfils the Equation (7) once putting $\Delta \mathrm{l}_{\text {Schw }} \equiv \Delta \mathrm{r}_{\mathrm{u}}$. On the one hand is seemingly surprising an expanding universe regarded as a super massive black hole; on the other hand the previous way to introduce Equation (7) does not make reference to any shrinking process, which therefore does not appear as a distinctive condition to allow a black hole. In effect appears arbitrary to extrapolate to the whole universe an idea usually acknowledged for observable events occurring inside the universe. The physics of the black hole foresees two important features: the existence of a central singularity and the so called "noodle effect" for an object fallen inside it. As concerns the former point, note that our physical universe is actually a hyper spherical shell defined by and corresponding to the uncertainty radial range $\Delta \mathrm{r}_{\mathrm{u}}=\mathrm{r}_{\mathrm{u}}-\mathrm{r}_{\mathrm{u} 0}$, whose boundaries are unknown and indefinable by fundamental assumption; indeed just $\Delta r_{u}$ along with its conjugate momentum range is consistent with the existence of physical observables. If $\mathrm{r}_{\mathrm{u} 0}<<\mathrm{t}_{\mathrm{u}}$, then the volume of the shell in practice approximates well numerically that of a global hyper sphere of radius $r_{u}$ centred on the origin of the reference system $\mathrm{R}$; in principle however the volume defined by $r_{u}$ includes a very small but finite internal volume $V_{u 0}$ of radius $r_{u}$, which has nothing to do with the physical properties inferable from $\Delta \mathrm{r}_{\mathrm{u}}$. Thus, once having introduced this latter $\mathrm{R}$ is replaced by any $\mathrm{R}^{*}$, in the frame of which hold the next considerations; moreover, whatever the time evolution of $\Delta r_{u}$ might be, one can admit that the singularity should fall within $\mathrm{V}_{\mathrm{u} 0}$ and thus in principle outside our physical universe. For the same reason this latter has no physical centre, i.e., the singularity does not affect its properties. As concerns the second point note that the noodle effect has been conceived for a compact body resulting by collapse of a larger object, whereas our universe is made by islands of matter surrounded by ample zones of vacuum. Is the latter kind of black hole physically similar to the dense usual one? Reasonably it does not, just because of the missing central singularity: such a black hole is presumably inconsistent with the aforesaid free fall towards a centre, which actually does not exist physically. Regard within $\Delta r_{u}$ any point of coordinate symbolized by $\mathrm{x}_{1}$ as a possible centre and recall that according to the shell theorem any test mass at the coordinate $\mathrm{x}_{2}$ is subjected to the force due to all material at smaller radius around $x_{1}$. As the range $x_{2}$ $\mathrm{x}_{1}$ falls entirely within $\Delta \mathrm{r}_{\mathrm{u}}$, hold the considerations of section 4.2 ; being both $\mathrm{m}$ and $\Delta \mathrm{l}$ therein introduced arbitrary, $\Delta \mathrm{l}$ can be identified with $\mathrm{x}_{2}-\mathrm{x}_{1}$ and $\mathrm{m}$ with the quoted smaller radius mass. There is no evidence about a noodle effect in this quantum model of black hole universe, which results compatible with that actually observed. Owing to the chance $r_{u 0}<<r_{u}$, in the following the volume of the universe will be estimated directly via $\Delta r_{u}$ rather than via $r_{u}$.

In the present model the basic idea is that of thinking a universe consistent with the condition of "maximum growth efficiency": this hypothesis seems reasonable for a growing universe, whose main requirement is to prevent mass and radiation energy losses outside it that could avert its possible evolution. According to the Hawking mechanism, based on the vacuum polarization in the presence of a strong gravity field, a black hole inside the universe splits a couple of virtual particles generated by vacuum quantum fluctuation; it captures one of them, while releasing the other that thus appears as an ordinary particle. This can be easily seen exploiting the previous results. Put $\Delta \mathrm{l} \equiv$ $\Delta \mathrm{l}_{\text {Schw }}$ into $\mathrm{U}=\mathrm{km}$ 'mG $/ \Delta \mathrm{l}$; it is trivial to infer $\mathrm{U}=$ $\mathrm{km}^{\prime} \mathrm{c}^{2} / 2$. This result concerns the energy of $\mathrm{m}$ ' just at the event horizon of the mass $\mathrm{m}$; moreover both $\mathrm{k}$ dependent values of $U$ involve $\mathrm{m}^{\prime}$ only. Recalling that $\mathrm{k}$ $= \pm 1$, it is possible to write $\mathrm{U}_{+}=\mathrm{m}^{\prime} \mathrm{c}^{2} / 2$ and $\mathrm{U}_{-}=$$\mathrm{m}^{\prime} \mathrm{c}^{2} / 2$; so $\Delta \mathrm{U}=\mathrm{U}_{+}-\mathrm{U}_{-}=\mathrm{m}^{\prime} \mathrm{c}^{2}$ and $\mathrm{U}_{+}+\mathrm{U}_{-}=0$. Clearly $U_{+}$and $U_{-}$regard two virtual particles of mass $\mathrm{m}^{\prime} / 2$ in its positive and negative energy states, whereas $\Delta U$ is the potential energy gap including the total energy of the particle-antiparticle pairs $\mathrm{m}^{\prime} \mathrm{c}^{2} / 2$ and $-\mathrm{m}^{\prime} \mathrm{c}^{2} / 2$; the masses of this system are independent on $\mathrm{m}$, they are however related to the black hole mass $\mathrm{m}$. This means that $\mathrm{m}$ ' could be the mass of a couple of charged virtual particles, which are therefore $\Delta \mathrm{l}_{\text {virt }}=\mathrm{e}^{2} / \Delta \mathrm{U}$ apart; hence it is possible that either of them is just at the event horizon of $\mathrm{m}$, whereas the other one is $\Delta \mathrm{l}_{\text {virt }}$ farther. So either particle of the system is captured by $\mathrm{m}$, the other one remains free to escape. Statistically both charges 
have the same probability of escaping, thus still contributing themselves to the virtual pairs that define the concept of "vacuum" of the universe. Outside the universe, however, this mechanism does not hold: the concept of vacuum, consisting of virtual particles continuously formed and annihilated, is replaced by that of "nothing". So no energy can escape outside $\Delta \mathrm{r}_{\mathrm{u}}$, which prevents useless waste of valuable energy content of the universe. In fact this assumption is directly related to the Hawking entropy and leads directly to the Friedmann equation. Before concerning these points let us concern again the basic consideration of maximum growth efficiency.

\subsection{The Black Hole Universe}

According to Equation (3), it must be also true that:

$$
\Delta \mathrm{p}_{\mathrm{x}}=\Delta \varepsilon \frac{\mathrm{v}_{\mathrm{x}}}{\mathrm{c}^{2}}
$$

In principle $\Delta \mathrm{p}_{\mathrm{x}}$ can be defined as $\mathrm{m}\left(\mathrm{v}_{\mathrm{x} 2}-\mathrm{v}_{\mathrm{x} 1}\right)$ or alternatively as $\Delta \mathrm{p}_{\mathrm{x}}=\mathrm{m}_{2} \mathrm{v}_{\mathrm{x}}-\mathrm{m}_{1} \mathrm{v}_{\mathrm{x}}$, being $\mathrm{m}_{1}$ and $\mathrm{m}_{2}$ arbitrary masses. The last form is more interesting for the purpose of the present section; multiplying both sides by $\mathrm{c}^{2} / \mathrm{v}_{\mathrm{x}}$, Equation (8) yields $\Delta \varepsilon+\mathrm{m}_{1} \mathrm{c}^{2}=\mathrm{m}_{2} \mathrm{c}^{2}$. This equation holds in general, i.e., whatever the masses and the energy range might represent. To specify this result, therefore, let us identify a particular physical meaning of these masses, as a function of which results also defined that of $\Delta \varepsilon$ by consequence. Specifying thus $m_{1}$ with the visible universe mass $m_{u}$ and putting $\mathrm{m}_{2}=\mathrm{M}_{\mathrm{u}}>\mathrm{m}_{\mathrm{u}}$, the last equation yields:

$$
\mathrm{M}_{\mathrm{u}} \mathrm{c}^{2}=\mathrm{m}_{\mathrm{u}} \mathrm{c}^{2}+\Delta \varepsilon
$$

i.e., $\Delta \varepsilon$ is an energy additional to that of the visible mass energy $\mathrm{m}_{\mathrm{u}} \mathrm{c}^{2}$. The physical meaning of this assignment, which defines a new greater mass $\mathrm{M}_{\mathrm{u}}$, can be verified exploiting known data of our universe. On the one hand, it is immediate to verify that Equation (7) written as $\Delta r_{u}=2 M_{u} G / c^{2}$ yields $M_{u}=10 m_{u}$. On the other hand, it is easy to check that holds the following equation defined by the today values of radius and age of the universe:

$$
\frac{\mathrm{M}_{\mathrm{u}}}{\mathrm{m}_{\mathrm{u}}} \frac{\mathrm{c} \Delta \mathrm{t}_{\mathrm{u}}}{\pi \Delta \mathrm{r}_{\mathrm{u}}} \approx 1 \quad \mathrm{M}_{\mathrm{u}}=10 \mathrm{~m}_{\mathrm{u}}
$$

Actually this result is inferred and extended to any time $\Delta \mathrm{t}$ in the quoted paper (Tosto, 2013); however for brevity this equation is simply introduced here without proof, but merely as a statement justified by the acknowledged radius and age of today's universe. Equation (10) supports the value of $\mathrm{M}_{\mathrm{u}}$ estimated via the Equation (7): It seems indeed significant that very large numbers fulfil such a simple numerical result. It also necessarily follows from Equation (9) that:

$\mathrm{M}_{\mathrm{u}}=\mathrm{m}_{\mathrm{u}}+\mathrm{m}_{?}$

An analogous conclusion would be obtained reasoning on the energy range. The notation of the second addend emphasizes the unknown nature of the additional mass $\mathrm{m}_{\text {? }}$, which is however required in the present model based on ranges of dynamical variables expressing the concept of uncertainty. Equation (11) is soon justified showing preliminarily that $\mathrm{M}_{\mathrm{u}}$ is reasonable; the next section 8 will concerns more explicitly $m_{\text {? }}$ itself. Let us show that the idea of supermassive black hole universe is supported itself by the following simple considerations.

\subsection{Hawking Entropy}

The first direct consequence of the idea of maximum growth efficiency is the Hawking entropy. Define first the circular frequencies of a light wave trapped by gravity around the border of the universe as $\omega_{\mathrm{n}}=\mathrm{n} \omega_{\mathrm{u}}$ being $\omega_{\mathrm{u}}=\mathrm{c} /\left(2 \pi \Delta \mathrm{r}_{\mathrm{u}}\right)$ (Tosto, 2013); so the boundary layer of the universe is marked out by the allowed frequencies of the electromagnetic field surrounding the total mass $\mathrm{M}_{\mathrm{u}}$ whose energy $\varepsilon_{\omega}$ is given by $\varepsilon_{\omega}=\mathrm{n} \hbar \mathrm{c} /\left(2 \pi \Delta \mathrm{r}_{\mathrm{u}}\right)$. Then let us concern also the total energy $\varepsilon_{\mathrm{u}}=\mathrm{M}_{\mathrm{u}} \mathrm{c}^{2}$ due to the whole amount of mass present in the universe. It is reasonable to expect that bulk energy $\varepsilon_{\mathrm{u}}$ and surface energy $\varepsilon_{\omega}$ should be someway correlated; so the simplest hypothesis is to introduce a dimensionless proportionality factor $\sigma_{\mathrm{H}}$ such that $\varepsilon_{\mathrm{u}}=\sigma_{\mathrm{H}} \varepsilon_{\omega}$. To infer the physical meaning of $\sigma_{\mathrm{H}}$, calculate the mean values of this equation, which reads $<\varepsilon_{\mathrm{u}}>=\sigma_{\mathrm{H}}<\varepsilon_{\omega}>$. Clearly $<\varepsilon_{\mathrm{u}}>\equiv$ $\varepsilon_{\mathrm{u}}$. The standard way to calculate $\langle\mathrm{n} \hbar \omega\rangle$ via the partition function is well known; noting that $\hbar \omega_{\mathrm{n}}<<\mathrm{k}_{\mathrm{B}} \mathrm{T}$ is verified for $\mathrm{T}$ down to values of the order of $10^{-28} \mathrm{~K}$, one finds $<\mathrm{n} \hbar \omega_{\mathrm{n}}>\approx \hbar \omega_{\mathrm{u}} \approx \mathrm{k}_{\mathrm{B}} \mathrm{T}$. So $\mathrm{k}_{\mathrm{B}} \sigma_{\mathrm{H}}$ defined by an energy over a temperature can be nothing else but entropy. With the help of the Plank length $1_{\mathrm{P}}=\sqrt{\hbar \mathrm{G} / \mathrm{c}^{3}}$, one finds therefore: 


$$
\begin{aligned}
& \sigma_{\mathrm{H}}=\frac{<\varepsilon_{\mathrm{u}}>}{<\mathrm{n} \hbar \omega_{\mathrm{u}}>}=\frac{\mathrm{A}_{\mathrm{u}}}{4 \mathrm{l}_{\mathrm{P}}^{2}} \hbar \omega_{\mathrm{u}}=\frac{\hbar \mathrm{c}}{2 \pi \Delta \mathrm{r}_{\mathrm{u}}} \\
& \varepsilon_{\mathrm{u}}=\frac{\mathrm{c}^{4}}{\mathrm{G}} \frac{\Delta \mathrm{r}_{\mathrm{u}}}{2} \mathrm{~A}_{\mathrm{u}}=4 \pi \Delta \mathrm{r}_{\mathrm{u}}^{2}
\end{aligned}
$$

In effect, $\sigma_{H}$ coincides just with the well known Hawking surface entropy in Boltzmann's units. Moreover, with the previous definition of $\omega_{\mathrm{u}}$, one also obtains with the help once more of Equation (7) the surface temperature $\mathrm{T}_{\mathrm{H}}$ of the black hole of mass $\mathrm{M}_{\mathrm{u}}$ :

$$
\mathrm{k}_{\mathrm{B}} \mathrm{T}_{\mathrm{H}}=\frac{\hbar \mathrm{c}^{3}}{4 \pi \mathrm{M}_{\mathrm{u}} \mathrm{G}}
$$

\subsection{Friedmann Solutions}

Consider an hyper sphere of volume $V_{u}=4 \pi \Delta r_{u}^{3} / 3$; this choice of $V_{u}$ is consistent with that of $A_{u}$ appearing in the Hawking entropy. Simply with the help of Equation (7) this volume reads $V_{u}=8 \pi \Delta r_{u}^{2} M_{u} G / 3 c^{2}$, which allows to calculate the average density of the universe as $\rho_{\mathrm{u}}=\mathrm{M}_{\mathrm{u}} / \mathrm{Vu}=3\left(\mathrm{c} / \Delta \mathrm{r}_{\mathrm{u}}\right)^{2} / 8 \pi \mathrm{G}$. Since $\mathrm{c} / \Delta \mathrm{r}_{\mathrm{u}}$ has physical dimensions $\operatorname{timr}^{-1}$, it can be surely expressed as $c / \Delta r_{u}=(a+b) / a$ with a variable length and b constant. The physical meaning of this position and in particular of a has been explained in the quoted paper (Tosto, 2013) with reference to the idea that $\Delta \mathrm{r}_{\mathrm{u}}{ }^{-1}$ represents a curvature. Here it is worth emphasizing that the presence of $b$ allows a non-vanishing curvature even for constant $\Delta r_{u}$ and a. So the hypothesis of black hole universe allows concluding:

$$
\frac{\rho_{\mathrm{u}}}{\rho_{\mathrm{c}}}=1+\frac{\mathrm{b}}{\dot{\mathrm{a}}}\left(2+\frac{\mathrm{b}}{\dot{\mathrm{a}}}\right) \rho_{\mathrm{c}}=\frac{3 \mathrm{H}^{2}}{8 \pi \mathrm{G}} \mathrm{H}=\frac{\dot{\mathrm{a}}}{\mathrm{a}}
$$

where, $\rho_{\mathrm{c}}$ is the well known critical density. This result is consistent with the solution of the Friedmann equations with cosmological constant different from zero; indeed replacing $c / \Delta r_{u}$ into the equation of $V_{u}$ one finds, putting $\mathrm{b}= \pm \mathrm{c}$ :

$$
\frac{8 \pi \mathrm{G} \rho_{\mathrm{u}}}{3}=\left(\frac{\mathrm{c}}{\mathrm{a}}\right)^{2}+\left(\frac{\dot{\mathrm{a}}}{\mathrm{a}}\right)^{2} \pm \frac{2 \mathrm{ca}}{\mathrm{a}^{2}}
$$

which compares well with the Friedmann equation including the cosmological constant $\Lambda$ once taking into account also the case of imaginary curvature with ia Equation (15):

$$
\frac{8 \pi \mathrm{G} \rho}{3}=\mathrm{k}\left(\frac{\mathrm{c}}{\mathrm{a}}\right)^{2}+\left(\frac{\dot{\mathrm{a}}}{\mathrm{a}}\right)^{2}-\frac{\Lambda \mathrm{c}^{2}}{3} \Lambda=\mp \frac{6 \dot{\mathrm{a}}}{\mathrm{ca}^{2}} \mathrm{k}= \pm 1
$$

It is worth noticing once again that also this solution of the Friedmann equation and its well known implications too, take here quantum character.

\subsection{Quantum Fluctuations}

Implement the Equation (1) to describe how changes a system during a quantum fluctuation. From a mathematical point of view, also this topic has been already described in the quoted paper (Tosto, 2013); here we concern the matter from a merely intuitive point of view, to emphasize how the fluctuation affects the behaviour of the system. Altering energies and momenta of the particles of any quantum system means that the initial uncertainty ranges sizes $\Delta \varepsilon$ and $\Delta p$ including the respective local values must be replaced by new ones $\Delta \varepsilon_{\mathrm{fl}}$ and $\Delta \mathrm{p}_{\mathrm{fl}}$ able to include also the altered values of dynamical variables during the corresponding time range $\Delta \mathrm{t}_{\mathrm{fl}}$; accordingly $\mathrm{n}_{\mathrm{fl}}$ is the related number of states during $\Delta \mathrm{t}_{\mathrm{fl}}$. By consequence change in particular all space ranges $\Delta \mathrm{x}$ including the space coordinates of the particles, in agreement with the obvious idea that also these latter are perturbed by the fluctuation. In principle it is reasonable to expect that by effect of the extra energy transient lasting $\Delta \mathrm{t}_{\mathrm{fl}}$ any particle of the system can be found where it couldn't before. In fact the Equation (1) represent a link between energy, momentum and space coordinates at any time; so it is not surprising that changing $\Delta \varepsilon$ and $\Delta \mathrm{p}_{\mathrm{x}}$ at any $\Delta \mathrm{t}$ affects by necessity all $\Delta \mathrm{x}$, i.e., expectedly $\Delta \mathrm{x}_{\mathrm{fl}}>\Delta \mathrm{x}$. By definition of uncertainty, however, what happens inside any delocalization range is in fact conceptually inaccessible to our knowledge and to any experimental measurement; ratios of range sizes like $\Delta \mathrm{x} / \Delta \mathrm{t}$ yield an average value only, related to the ability of the particle to travel throughout $\Delta \mathrm{x}$ during $\Delta \mathrm{t}$. Actually neither the former nor the latter take assigned values, being the uncertainty range sizes arbitrary and unknowable by definition; so $\mathrm{V}$ is arbitrary and never associated to an assigned value, the only assigned and measurable value being in this model c. Hence any speculation about how fast the particles move or displace within $\Delta \mathrm{x}_{\mathrm{fl}}$ during $\Delta \mathrm{t}_{\mathrm{fl}}$ is unphysical; nevertheless it is shown in (Tosto, 2013) that the fluctuation driven $\Delta \mathrm{x}_{\mathrm{fl}}>\Delta \mathrm{x}$ allows the particles to be found where they shouldn't according to the classical physics. What is indeed surprising is that the fluctuation driven swelling of the space ranges, related to strong 
momentum and energy changes, corresponds to displacements of the particles even compatible with superluminal velocities. Elementary algebraic considerations show indeed a further weirdness of the quantum world, i.e., that $\mathrm{v}_{\mathrm{fl}}=\Delta \mathrm{l}_{\mathrm{fl}} / \Delta \mathrm{t}_{\mathrm{fl}}=\mathrm{c}^{2} / \mathrm{v}$, whereas instead $\Delta \mathrm{l} / \Delta \mathrm{t}<\mathrm{c}$ before the fluctuation. Actually, owing to the character intrinsically arbitrary of $\mathrm{v}$ and $\mathrm{v}_{\mathrm{fl}}$, is more appropriate to say that the particles spread during the fluctuation throughout ranges sized as if they would have superluminal velocity. Nevertheless $\mathrm{v}_{\mathrm{fl}}>\mathrm{c}$ holds just because $\mathrm{v}<\mathrm{c}$, whereas for light particles in vacuum for which $\mathrm{v} \equiv \mathrm{c}$ one always finds invariably $\mathrm{v}_{\mathrm{fl}}=\mathrm{c}$.

These conclusions are of interest to describe the inflationary era of our universe, in particular during the transition from radiation era to matter era. Consider a growing hyper sphere approaching to the end of the radiation era: it contains energetic light particles together with fermion matter particles formed by high energy photon-photon interactions (Moffat, 1993; Walsh and Zerwas, 1973). If the growth of the sphere is still within the time range $\Delta \mathrm{t}_{\mathrm{fl}}$, as it is reasonably possible to expect during the first radiation era after the birth of the universe, the matter particles displace farther than the light itself; so, according to this reasoning, the light should remain preferentially confined within an inner shell that serves as energy reservoir for the further formation of matter allowed to spread towards an outer shell. Of course it is conceptually impossible to calculate or estimate how long lasts $\Delta \mathrm{t}_{\mathrm{fl}}$; the reason is conceptually analogous to that preventing the possibility of predicting exactly when a radioactive atom decays. Nonetheless, it is possible to recognize three steps of growth of the new born universe: (i) the initial one before the beginning of the fluctuation; (ii) that during $\Delta \mathrm{t}_{\mathrm{fl}}$ controlled by the fluctuation extra-energy transient during which the global growth was superluminal-like and (iii) that successive to $\Delta \mathrm{t}_{\mathrm{fl}}$ controlled by the standard expansion rate slower than the light speed.

\subsection{Possible Timeline of the Universe}

Consider a quantum bubble having diameter of the order of the Planck length and containing energy equivalent to all mass of the early universe. It is known that if such bubble would be created from nothing, it would soon collapse because of its own gravitational field and would turn into a singularity. According to the short discussion of section 4.2, however, both gravity and anti-gravity are possible outcomes as a consequence of Equation (1) because of $\mathrm{k}= \pm 1$. So, why not to think that the anti-gravity anticipated the gravity? The possible early steps should be therefore: (i) the initial growth dynamics of the quantum bubble was governed by the repulsive effect of anti-gravity with $\Delta \mathrm{l}$ at the Planck scale $1_{\mathrm{Pl}}$, so that the results of sections 4.1 and 4.2 allow writing $U$ $=\varepsilon^{\prime} \varepsilon \mathrm{G} /\left(\mathrm{c}^{4} l_{\mathrm{P}}\right)$ with $\varepsilon^{\prime}=\hbar \omega^{\prime}$ and $\varepsilon=\hbar \omega$; (ii) the energy gap $2 U=2 \varepsilon^{\prime} \varepsilon G /\left(c^{4} \Delta \mathrm{l}\right)$ due to decay of anti-gravity to gravity reasonably occurred late enough to allow $\Delta \mathrm{l}>\mathrm{l}_{\mathrm{Pl}}$ and prevent the bubble from collapsing, despite the new born attractive effect inevitably slowed down its initial explosive boost; (iii) the occurring of the quantum fluctuation then further revitalized the expansion of the universe against the attractive effect of gravity force; (iv) the arising of further kinds of interaction forces additional to and subsequent the gravity was next necessary to manage the charges created by the intense light radiation field expected during the step (i); (v) followed then the dust era, characterized by the presence of matter originated during the expansion driven cooling of the radiation field. Note that in this qualitative sketch the radiation era started first as a result of the decay energy $2 \mathrm{U}=$ $\varepsilon^{\prime} \varepsilon \mathrm{G} /\left(\mathrm{c}^{4} l_{\mathrm{P}}\right)$; so $\varepsilon^{\prime} \varepsilon=\hbar^{2} \omega^{\prime} \omega$ corresponds to the photonphoton interaction, which results to be a higher order effect according to $\hbar^{2}$. Clearly $c^{4} l_{\mathrm{P}} / \mathrm{G}$ is the expected Planck energy. The formation of matter was allowed when the fluctuation energy propelled away the early virtual pairs towards a colder external shell surrounding the hot light radiation volume. As the enhanced expansion rate contributed to the formation of matter, it is not surprising that the separation of strong and electroweak interactions from the gravity was activated just at the beginning of the fluctuation driven inflationary era. The Fig. 1 sketches qualitatively a possible timeline of the early universe.

\subsection{The Missing Mass}

The idea of a missing mass additional to the visible mass $\mathrm{m}_{\mathrm{u}}$ is supported by Equation (9) and (11) consequence of Equation (10). In effect the hypothesis of black hole universe requires $m_{\text {? }}$ in order to define $M_{u}$ that fulfils the condition (7). It is interesting to rewrite identically the Equation (10) as follows:

$$
\mathrm{m}_{\mathrm{u}} \mathrm{c}^{2} \hbar / \Delta \mathrm{t}_{\mathrm{u}}=\mathrm{m}_{\mathrm{u}} \mathrm{c}^{2} \hbar \omega_{\mathrm{u}}+\mathrm{m}_{?} \mathrm{c}^{2} \hbar \omega_{\mathrm{u}} \omega_{\mathrm{u}}=\frac{\mathrm{c}}{\pi \Delta \mathrm{r}_{\mathrm{u}}}
$$




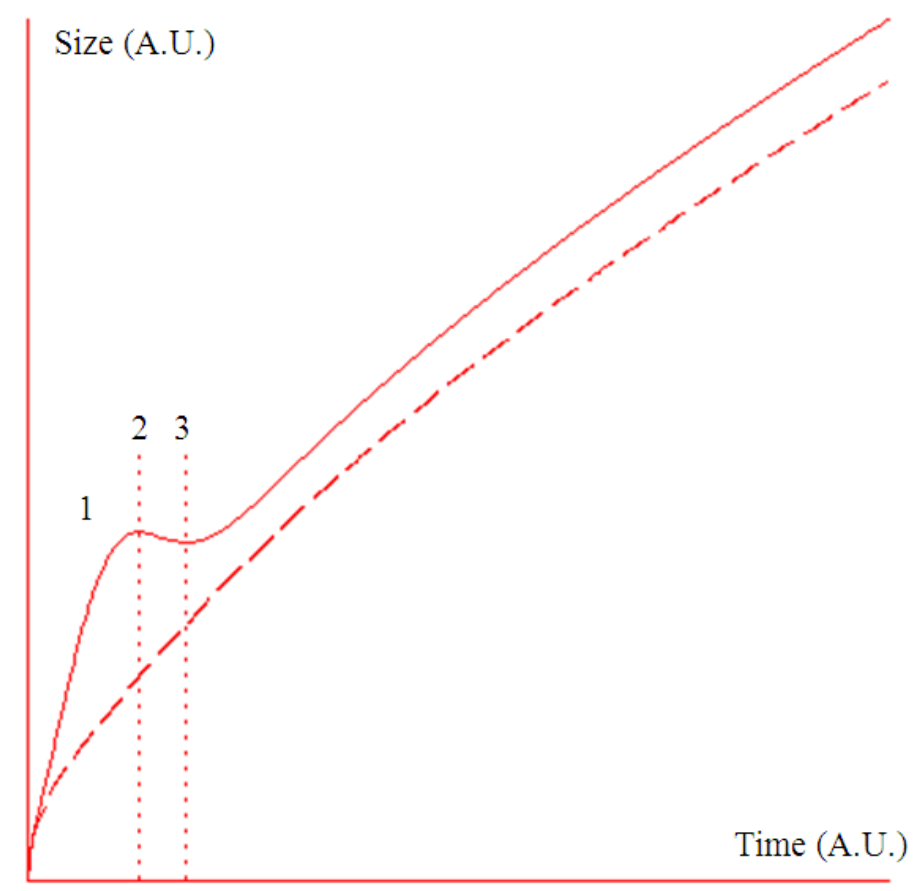

Fig. 1. Schematic timeline of the universe expansion; the space and time scales are in arbitrary dimensionless units. The time range 1 shows the anti-gravity stage corresponding to $U=\hbar^{2} \omega^{\prime} \omega / E_{P}$ at the Planck era. At the time 2 starts the anti-gravity $\rightarrow$ gravity decay; the abrupt arising of attractive Newtonian gravity slows down the expansion, qualitatively emphasized here by some size contraction till to the time 3 . At this time starts the fluctuation that triggers the inflationary era and corroborates the expansion; the increased size as a function of time is boosted by the extra-energy transient of the fluctuation. The dashed line represents a plain combination of $\mathrm{t}^{1 / 2}$ and $\mathrm{t}^{2 / 3}$ time profiles before and after the inflationary era

To emphasize that the mass $m_{\text {? }}$ results strictly interrelated to the visible mass $m_{u}$ and to the frequency $\omega_{\mathfrak{u}}$ yielding the Hawking entropy. This motivates rationally $\mathrm{m}_{?}$. This equation is defined by a sum of square energies; regarded as $\varepsilon_{\text {tot }}^{2}=\varepsilon_{\text {kin }}^{2}+\varepsilon_{\text {rest }}^{2}$, the form of Equation (16) is similar to that of Equation (5), with $\mathrm{m}_{\mathrm{u}} \mathrm{c}^{2} \hbar \omega$ corresponding to the kinetic term $(\mathrm{pc})^{2}$ and $\mathrm{m}_{2} \mathrm{c}^{2} \hbar \omega$ to the rest term $\left(\mathrm{mc}^{2}\right)^{2}$. This comparison suggests therefore that $m_{\text {? }}$ could be related to the rest energy of the universe, which in turn requires that the universe as a whole should also move as if it would be a spinless and chargeless free macro-particle. In principle this idea does not conflict with any experimental fact detectable in our universe. An observer at rest in a reference system $R^{\prime}$ could not discern whether its $R^{\prime}$ 'is at rest or moves at constant velocity with respect to the origin of another reference system R; so we cannot notice whether or not our whole universe is at rest or animated by uniform motion in any external $\mathrm{R}$.
On the one hand, the basic idea is reasonable: once having introduced in Equation (12) and (13) an hyper sphere representing the whole universe, why not to consider the chance of whether this hyper sphere moves or not? Clearly the answer requires a reference system external to our universe with respect to which the question has physical meaning itself. However, it has been emphasized that the physical properties of the universe depend on the uncertainty ranges $\Delta \mathrm{t}_{\mathrm{u}}$ and $\Delta \mathrm{r}_{\mathrm{u}}$; if so however we have emphasized in section 2 that any reference system is compatible with the quantum approach based on Equation (1) only, through which are to be inferred the key quantum features of the universe; so the previous question, in principle correct from a physical point of view, can be left in practice unanswered because inessential to infer the quantum eigenvalues and thus the observables themselves. In effect it is shown in (Tosto, 2013) how to handle mathematically Equation (16) in order to regard it not only at the present $\Delta \mathrm{t}_{\mathrm{u}}$ but, more in general, at any time $\Delta t$; here we report this significant conclusion only. 
The rest mass è of the universe related to $\mathrm{m}_{\text {? }}$ includes the masses of all invisible dust, all invisible black holes and surface energy of the universe to which corresponds the Hawking entropy plus an energy times $\mathrm{c}^{-2}$ required for a total balance $\varepsilon_{\text {rest }}$; in turn all these contributions, related to $\pm \sqrt{\mathrm{m}_{?} \mathrm{c}^{2} \hbar \omega}$, correspond to the rest energy of a free macro-particle of constant mass moving at constant velocity. The fact that the "dark energy" is dark, is no longer surprising: it does not depend on something existing inside the universe, it concerns the whole universe regardless of any kind of mass visible or not contributing to $\mathrm{M}_{\mathrm{u}}$.

\section{DISCUSSION}

The previous sections have highlighted the basic ingredients of the present model of quantum universe: the antigravity, the quantum fluctuation, the Friedmannlike equation and the black hole critical mass achieved with the help of an additional dark mass. The first and most important remark is the lack of classical determinism in the quantum formulae here obtained as corollaries of the fundamental Equation (1) only. This aspect has been emphasized when commenting the quantum character of Equation (3) and (5); their relativistic familiarity should not mask their profound difference from the early Einstein formulas: these latter could not be implemented to describe energy and momentum quantum fluctuations. This holds also for the steps of section 7 . There is no reason to require that the history of the universe is just exactly that therein sketched; small differences from that scheme would have accordingly implied a universe slightly different from our own or even no universe at all. The best way to justify the assumptions of section 7 rests on the actual existence of the universe itself. Eventually, these considerations hold also for the Friedmann equation (14): all conclusions about critical density as crucial criterion to determine the ultimate evolution and fate of the universe must be regarded from the standpoint of the quantum character of this equation too. Ample debate is reported in literature about the chance that the density parameter $\Omega$ is greater or equal or lower than 1 ; however Equation (14) is obviously not deterministic. The reasoning recalls the thought experiment of the Schrodinger cat, which is made by a combination of wave functions dead cat and living cat. This superposition of states opens both chances, either of which effectively happens when the total wave function collapses into the respective chance for the cat by consequence of the observation process. The so called "multi world history" hypothesis was just inspired by extrapolating both chances opened by the interaction observer-cat to all events possible in the history of the mankind. This analogy suggests that actually the wave function of the whole universe could be a superposition of states with $\Omega=-1, \Omega=0$ and $\Omega=1$, so that in principle all respective consequences would be probabilistically allowed. Otherwise stated, searching specifically one only among these chances in a deterministic way is an attempt that, according to the quantum theory, is actually unphysical. The pure relativistic approach followed by Friedmann when solving the Einstein field equation, skips and therefore misses the indeterminism of the quantum theory. In conclusion: the search of a theory taking into account a better estimate of the Hubble constant or actual density of the universe to decide a unique final fate of our universe, whatever it might be, seems inappropriate and misleading. This conclusion is in fact not surprising once accepting the idea that the behaviour of nature follows the space-time uncertainty, which per se is inconsistent with the idea of a unique timeline rigidly fixed after the big bang. As a matter of fact, however, Equation (1) yield sensible consequences. Further activity is in progress to better investigate the present results.

\section{CONCLUSION}

Equation (1) appear adequate to formulate a quantum model of universe for two reasons: on the one hand, the arbitrariness of the uncertainty ranges allows specifying them according to the physical meaning of any particular problem; on the other hand, the conceptual lack of information and constrains on the range sizes makes them suitable to describe nano-, micro- and macro-scale effects. These features are in effect essential and required to bridge quantum mechanics and relativity; as the cosmology is intrinsically made of both, the space-time uncertainty appears to be a formidable tool to tackle cosmological problems.

\section{REFERENCES}

Adams, F.C. and G. Laughlin, 1997. A dying universe: The long-term fate and evolution of astrophysical objects. Rev. Modern Phys., 69: 337-372. DOI: 10.1103/RevModPhys.69.337 
Bertone, G., D. Hooper and J. Silk, 2005. Particle dark matter: Evidence, candidates and constraints. Phys. Rep., 405: 279-390. DOI: 10.1016/j.physrep.2004.08.031

Burbridge, E.M., G.R. Burbridge, W.A. Fowler and F. Hoyle, 1957. Synthesis of the elements in stars. Rev. Mod. Phys., 29: 547-650. DOI: 10.1103/RevModPhys.29.547

Canetti, L., M. Drewes and M. Shaposhnikov, 2012. Matter and antimatter in the universe. New J. Phys., 14: 095012-095012. DOI: $10.1088 / 1367-$ 2630/14/9/095012

Carroll, S.M. and J. Chen, 2004. Spontaneous inflation and the origin of the arrow of time. Cornell University Library.

Davies, P.C.W., 1978. thermodynamics of black holes. Rep. Prog. Phys., 41: 1313-1355. DOI: 10.1088/0034-4885/41/8/004

Dine, M. and A. Kusenko, 2003. The origin of the matter-antimatter asymmetry. Cornell University Library.

Dolgov, A.D., 2002. Cosmological matter-antimatter asymmetry and antimatter in the universe. Cornell University Library.

Gupta, S.N., 1962. Quantum Theory of Gravitation, Recent Developments in General Relativity. 1st Edn., Pergamon Press, pp: 258.

Hawking, S.W. and R. Penrose, 1970. The singularities of gravitational collapse and cosmology. Proc. R Soc. A, 314: 529-548. DOI: 10.1098/rspa.1970.0021

Islam, J.N., 1977. Possible ultimate fate of the universe. Q. J. Royal Astronom. Soc., 18: 3-8.

Krauss, M. and G.D. Starkman, 2000. Life, the universe and nothing: Life and death in an ever-expanding universe. Lawrence Astrophys. J., 531: 22-30. DOI: 10.1086/308434

Kroupa, P., 2010. Local-group tests of dark-matter concordance cosmology: Towards a new paradigm for structure formation. Astron. Astrophys., 523: 32-54. DOI: 10.1051/0004-6361/201014892
Liddle, A.R. and D.H. Lyth, 2000. Cosmological Inflation and Large-Scale Structure. 1st Edn., Cambridge University Press, Cambridge, ISBN-10: 0521575982, pp: 400.

Moffat, J.W., 1993. Superluminary universe: A possible solution to the initial value problem in cosmology. Int. J. Mod. Phys. D, 2: 351-365. DOI: 10.1142/S0218271893000246

Penrose, R., 1965. Gravitational collapse and space-time singularities. Phys. Rev. Lett., 14: 57-59. DOI: 10.1103/PhysRevLett.14.57

Tosto, S., 1996a. An analysis of the states in the phase space: The energy levels of quantum systems. Il Nuovo Cimento B, 111: 193-215. DOI: 10.1007/BF02724645

Tosto, S., 1996b. An analysis of states in the phase space: The diatomic molecules. Il Nuovo Cimento D, 18: 1363-1394. DOI: 10.1007/BF02453780

Tosto, S., 2012. Quantum uncertainty and relativity. Prog. Phys., 2: 58-81.

Tosto, S., 2013. Space-time uncertainty and cosmology: A proposed quantum model of the universe. Prog. Phys., 4: 3-24.

Trimble, V., 1987. Existence and nature of dark matter in the universe. Ann. Rev. Astron. Astrophys., 25: 425-472.

DOI: 10.1146/annurev.aa.25.090187.002233

Walsh, T.F. and P. Zerwas, 1973. Two-photon processes in the parton model. Phys. Lett. B, 44: 195-198. DOI: 10.1016/0370-2693(73)90520-0

Weinberg, S., 1996. The Quantum Theory of Fields II: Modern Applications. Cambridge University Press.

Zhi-Zhong, X., 2007. Cosmological matter-antimatter asymmetry and neutrino oscillations. Nucl. Phys. B, 166: 30-34. DOI: 10.1016/j.nuclphysbps.2006.12.005 